IZA DP No. 4178

Coming to America:

Does Immigrant's Home Country Economic Status Impact the Probability of Self-Employment in the U.S.?

Ruth Uwaifo Oyelere

Willie Belton

May 2009 


\title{
Coming to America: Does Immigrant's Home Country Economic Status Impact the Probability of Self-Employment in the U.S.?
}

\author{
Ruth Uwaifo Oyelere \\ Georgia Institute of Technology \\ and IZA \\ Willie Belton \\ Georgia Institute of Technology
}

Discussion Paper No. 4178

May 2009

IZA

P.O. Box 7240

53072 Bonn

Germany

Phone: +49-228-3894-0

Fax: +49-228-3894-180

E-mail: iza@iza.org

Any opinions expressed here are those of the author(s) and not those of IZA. Research published in this series may include views on policy, but the institute itself takes no institutional policy positions.

The Institute for the Study of Labor (IZA) in Bonn is a local and virtual international research center and a place of communication between science, politics and business. IZA is an independent nonprofit organization supported by Deutsche Post Foundation. The center is associated with the University of Bonn and offers a stimulating research environment through its international network, workshops and conferences, data service, project support, research visits and doctoral program. IZA engages in (i) original and internationally competitive research in all fields of labor economics, (ii) development of policy concepts, and (iii) dissemination of research results and concepts to the interested public.

IZA Discussion Papers often represent preliminary work and are circulated to encourage discussion. Citation of such a paper should account for its provisional character. A revised version may be available directly from the author. 


\section{ABSTRACT \\ Coming to America: \\ Does Immigrant's Home Country Economic Status Impact the Probability of Self-Employment in the U.S.?*}

This paper examines the impact of home country economic status on immigrant selfemployment probability in the U.S. We estimate a probability model and find that, consistent across race, immigrants from developed countries are more likely to be self-employed in the U.S than are immigrants from developing countries. This result is unexpected given previous research which suggests that immigrants from countries with high levels of self-employment tend to be more involved in self-employment in the U.S. Developing countries on average have higher self-employment rates than do developed countries but our research shows that immigrants from developing countries have similar or lower self-employment probabilities relative to native born White Americans, whereas immigrant from developed countries have significantly higher self-employment probabilities relative to native born White Americans. We provide two potential explanations for this result. First, immigrants from developed countries may indeed have more and better access to start-up capital from their country of origin. Second, institutional arrangements in the developed world may be similar across countries allowing immigrants from developed countries to have an informational advantage over immigrants from developing countries.

JEL Classification: J21, E24, J61, J40

Keywords: $\quad$ self-employment, immigrant, home country, entrepreneurship

Corresponding author:

Ruth Uwaifo Oyelere

Georgia Institute of Technology

School of Economics

221 Bobby Dodd Way

Atlanta, GA 30332-0615

USA

E-mail: ruth.uwaifo@econ.gatech.edu 


\section{Introduction and Background}

Self-employment in the United State has been of interest to policy-makers for a number of reasons: First, many believe that self-employment provides a time tested method for recent immigrants, minorities, and other under-represented groups to break the poverty cycle allowing entry into mainstream U.S. economic life. Second, since immigrations has been responsible for the lion-share of U.S. population growth of the last 30 years, many are concerned about the impact of increased immigrant population on the country's ability to provide social and economic services to its' population. Historically, immigrants were thought to be predisposed to self-employment providing additional employment opportunities for themselves and many other Americans. Briggs and Moore (1994) argued that the distinguishing characteristic of immigrants is their very high levels of entrepreneurship. Since immigrants were thought to create more benefits than costs even conservative commentator Joel Kotkin (Kotkin 1991) argued that immigration should be encouraged.

Yuengert (1995) and Fairlie and Meyer (1996) provide the most recent research that explicitly investigates the role of home country attributes in the immigrant's self-employment decision in the U.S. Based on home country attributes, Yuengert (1995) finds that immigrants from countries with high self-employment rates relative to U.S. rates tend to pursue self-employment at a higher rate than do natives. He argues that experiences in the informal sector of the home country is a form of sector-specific human capital inclining immigrants more toward self-employment in the United States. This process could work through increased immigrant efficiency in starting up and/or managing new businesses venture or immigrants could have more human and/or financial capital to invest in a self-employment venture (See Bogan and Darity 2008). Fairlie and Meyers (1996) argue that we need to be more cautious about this conclusion. They find that if the probability model is estimated removing natives from the data set, that self-employment rate of the home country does not have a significant statistical impact on an ethnic group's self-employment probability in the U.S.

In this research we attempt to further investigate the home country hypothesis by examining the impact of home country economic standing on the probability of self-employment of U.S. immigrants. We distinguish home country economic standing by dividing all countries into two groups; developed 
and developing countries. From a financial and education resources availability perspective, a priori, one could argue that immigrants from developed countries should, on average, be more prepared to engage in self-employment than are their developing nation counterparts. However, Yuengert (1995) finds that immigrants from countries where the percent of the labor force that identifies as self-employed is large relative to the U.S. percentage, are more likely to seek self-employment in the United States. Consulting the Yearbook of Labor Statistics (2005), we examine a subset of immigrant home countries including those of Latin and South America, Asia, eastern and western Europe and Africa. We find that countries with self-employment rates that are significantly higher than that of the U.S. are generally those that are identified by the World Bank and the IMF (International Monetary Fund) as developing or less developed countries. Given this finding, the implication of Yuengert's work is that after immigrating to the U.S., immigrants from developing countries are more likely to pursue self-employment than are immigrants from developed countries. In what follows we carefully investigate the impact of home country economic standing on self-employment probabilities for U.S. immigrants.

First, we partition the sample based on race and country of birth. For those born outside the U.S., we break down the sample based on citizenship and home country's economic status. Using logit and probit models, we estimate the probability of self-employment for all groups relative to U.S. born White Americans our base comparison group. We find that foreigners from developing countries have lower self-employment probabilities than do U.S. born White Americans, while foreigners from developed countries have higher self-employment probabilities relative to U.S. born White Americans. Further, considering naturalized citizens relative to U.S. born White Americans we find that those from developing countries have lower self-employment probabilities than those from developed countries but similar or lower probabilities than U.S. born White Americans. In an attempt to examine the robustness of our result we follow Fairlie and Myers (1996) and re-estimate all models base on a sample of immigrants only. We find that immigrants from a developed countries have a higher probability of self-employment in the U.S. than do their developing country counterparts. Given the robustness of our results we provide two possible explanations for our empirical 
outcomes: First, immigrants from developed countries may indeed have more and better access to start-up capital from their country of origin and may find it easier to start a business. Second, institutional arrangements, business types, and business "know-how" may be similar across countries in the developed world. This could lead to an advantage for immigrants from developed countries with respect self-employment success in the U.S.

The rest of the paper is organized as follows. The second section provides an overview highlighting important attributes of the data set. Section 3 describes the econometric approach and, in a step by step format, outline the identification strategy, provide econometric results, and also offer robustness checks of key results. Sections 4 provides alternative explanations of our empirical results. The final section contains a discussion of inferences, conclusions, and recommendations.

\section{Data}

\subsection{General Description of Datasets}

We make use of the March Current Population Survey (CPS). The CPS is micro-data that provides information about individual persons and households. It is a monthly U.S. household survey conducted jointly by the U.S. Census Bureau and the Bureau of Labor Statistics. We derive multistage stratified samples of the CPS from IPUMS. The IPUMS-CPS data are available for 46 years (1962-2007). We use data from 1994-2002 because of the existence of important variables which, in many cases, were not surveyed until 1994. For example, parent's birthplace was not available in the CPS before 1994. Similarly, post 2002 the coding for race changed significantly as the variable that captures race was broken into several subcategories making it more difficult to easily identify groups of interest. Specifically, prior to 2003 the number of race categories ranged from 3 (White, Negro, and other) to 5 (White, Black, American Indian/Eskimo/Aleut, Asian or Pacific Islander, and other). The three-category breakdown of race was thought to be too simplistic and was abandoned in 1988 for the more empirically useful five category breakdown. Beginning in 2003, respondents could report more than one race, and the number of codes increased to 21 making it more difficult to compare racial identification prior to 2003 with post 2003. Individuals who previously self-identified 
as Black could now identify themselves as biracial, likewise, others who self-identified as White prior to this change could now claim multi-racial status. One of the advantages of using the CPS via IPUMS is that it makes cross-time comparisons using the March CPS data more feasible as variables in IPUMS-CPS are coded identically or "harmonized" for 1962 to 2007.

\subsection{Descriptive Statistics}

Table 1: Breakdown of Data by Racial Categories

\begin{tabular}{cccc}
\hline \hline & & & \\
Variable & Observations & $\%$ & Probability of self-employment \\
\hline Black US born & 127,617 & 9.77 & 0.043 \\
White US born & 998,205 & 76.40 & 0.119 \\
Black Naturalized & 3,374 & 0.26 & 0.068 \\
White Naturalized LDC & 22936 & 1.76 & 0.115 \\
White Naturalized DC & 8491 & 0.64 & 0.198 \\
Black Foreign & 6,163 & 0.47 & 0.051 \\
White Foreign LDC & 61,327 & 4.69 & 0.063 \\
White Foreign DC & 7,059 & 0.54 & 0.148 \\
American Indian/Aleut/Eskimo & 18,368 & 1.41 & 0.073 \\
Asian or Pacific Islander US born & 20,811 & 1.59 & 0.079 \\
Naturalized Asian or Pacific Islander LDC & 9473 & 0.72 & 0.124 \\
Naturalized Asian or Pacific Islander DC & 2164 & 0.17 & 0.249 \\
Foreign Asian or Pacific Islander LDC & 11,591 & 0.89 & 0.065 \\
Foreign Asian or Pacific Islander DC & 3,487 & 0.27 & 0.190 \\
Other (single) race & 5,579 & 0.43 & 0.057 \\
\hline \hline
\end{tabular}

Table (1) provides an overview of the data sample used in this research. The sample includes 1,296,606 records where $84.04 \%$ of respondents reported race as White, $10.4 \%$ as Black, $1.41 \%$ as American Indian, Aleut or Eskimo, $3.63 \%$ as Asians or Pacific Islanders, and $0.43 \%$ classify themselves as Other. This table provides a break-down of the data set into relevant subgroups. These subgroups are based on race and on citizenship status. We divide individuals into those who are U.S born, U.S. naturalized citizen, and non U.S. citizen. For individuals who are immigrants and/or naturalized citizens we partition the data by home country economic standing i.e., developed or 
developing. For each sub-groups we highlight its' share in the population and provide the estimated probability of U.S. self-employment. For example foreign Asians or Pacific Islanders from developed countries (DC) are $0.27 \%$ of the population and have a $19 \%$ probability of being self-employed. The groups with the highest probabilities of self-employment are naturalized Asians from developed countries followed by naturalized Whites from developed countries.

\subsection{Self-employment rates across countries}

The Yearbook of Labor Statistics provides the needed data for calculating each country's labor force share that selects self-employment as their major source of income and/or financial support. Table (2) provides available data, for each country, corresponding to the percent of each country's labor force which selects self-employment. The data reveals that countries which are categorized as developing nations, by IMF and World Bank standards, tend to on average have higher selfemployment percentages than do those that are listed as developed nations. However, it is also important to note that among both developing and developed countries, there is significant variation in self-employment percentages. Given the findings summarized in Table (2), and the literature which suggests that self-employment rates for immigrants to the U.S. should mimic home country self-employment rates, a priori, we would expect immigrants from developing countries to have higher probability of self-employment in the U.S. than do their developed country counterparts.

\section{$3 \quad$ Econometric Model and Results}

\subsection{General econometric model}

To investigate the impact of immigrant home country's economic status on the probability of selfemployment, we estimate a variants of equation (1) making use of three different empirical strategies. Equation (1) is a simple self-employment probability model:

$$
\operatorname{Prob}(Y=1)=F\left(\beta^{\prime} X\right)
$$

where $\mathrm{X}$ is a matrix of all the possible factors impacting the probability of self-employment. If $\mathrm{Y}=1$, an individual is self-employed, whereas $\mathrm{Y}=0$ indicates that an individual is a wage worker. 
Table 2: self-employment rates in select Developing and Developed Countries

\begin{tabular}{|c|c|c|c|c|c|c|c|}
\hline \multicolumn{4}{|c|}{ Developing Countries } & \multicolumn{4}{|c|}{ Developed Countries } \\
\hline Country & $\begin{array}{c}\text { source } \\
(1)\end{array}$ & $\begin{array}{c}\text { year } \\
(2)\end{array}$ & $\begin{array}{l}\text { Self Emp. Ratio } \\
(3)\end{array}$ & $\begin{array}{c}\text { Country } \\
(4)\end{array}$ & $\begin{array}{c}\text { source } \\
(5)\end{array}$ & $\begin{array}{l}\text { year } \\
(6)\end{array}$ & $\begin{array}{c}\text { Self Emp. Ratio } \\
(7)\end{array}$ \\
\hline Russian & LFS & 2004 & 0.076 & United Kingdom & $\overline{L F S}$ & 2003 & 0.039 \\
\hline Estonia & LFS & 2003 & 0.088 & Luxembourg & LFS & 2004 & 0.066 \\
\hline Macau, China & LFS & 2004 & 0.118 & Norway & LFS & 2004 & 0.0707 \\
\hline Slovakia & LFS & 2004 & 0.12 & United States & LFS & 2004 & 0.073 \\
\hline Ukraine & LFS & 2003 & 0.124 & France & LFS & 2004 & 0.116 \\
\hline Malta & LFS & 2004 & 0.137 & Netherlands & LFS & 2004 & 0.121 \\
\hline Hungary & LFS & 2004 & 0.15 & Finland & LFS & 2004 & 0.127 \\
\hline Bulgaria & LFS & 2004 & 0.152 & Australia & LFS & 2004 & 0.129 \\
\hline Slovenia & LFS & 2004 & 0.155 & Switzerland & LFS & 2004 & 0.133 \\
\hline Czech Republic & LFS & 2004 & 0.168 & New Zealand & LFS & 2004 & 0.135 \\
\hline Lithuania & LFS & 2004 & 0.186 & Israel & Unknown & 2004 & 0.144 \\
\hline Mauritius & Other & 2004 & 0.197 & Japan & LFS & 2004 & 0.148 \\
\hline Croatia & LFS & 2004 & 0.209 & Canada & LFS & 2004 & 0.154 \\
\hline Cyprus & LFS & 2004 & 0.237 & Spain & Unknown & 2004 & 0.193 \\
\hline Panama & LFS & 2004 & 0.265 & Ireland & Other & 2004 & 0.201 \\
\hline Macedonia & LFS & 2003 & 0.273 & Portugal & LFS & 2004 & 0.262 \\
\hline Argentina & LFS & 2004 & 0.279 & S. Korea, & Unknown & 2004 & 0.366 \\
\hline Uruguay & LFS & 2004 & 0.294 & Greece & LFS & 2005 & 0.375 \\
\hline Romania & LFS & 2004 & 0.341 & Average & & & $\underline{0.146}$ \\
\hline Indonesia & LFS & 2003 & 0.345 & & & & \\
\hline Moldova & Unknown & 2004 & 0.367 & & & & \\
\hline Pakistan & LFS & 2003 & 0.373 & & & & \\
\hline Honduras & LFS & 2001 & 0.388 & & & & \\
\hline Mexico & LFS & 2004 & 0.403 & & & & \\
\hline Azerbaijan & LFS & 2003 & 0.435 & & & & \\
\hline Sri Lanka & LFS & 2003 & 0.441 & & & & \\
\hline Guatemala & LFS & 2004 & 0.446 & & & & \\
\hline Ethiopia & LFS & 2004 & 0.495 & & & & \\
\hline Zimbabwe & LFS & 1999 & 0.538 & & & & \\
\hline Thailand & LFS & 2000 & 0.545 & & & & \\
\hline Georgia & LFS & 2003 & 0.659 & & & & \\
\hline Albania & Other & 2001 & 0.685 & & & & \\
\hline Bolivia & Other & 2000 & 0.704 & & & & \\
\hline Madagascar & LFS & 2001 & 0.815 & & & & \\
\hline Tanzania & LFS & 2000 & 0.931 & & & & \\
\hline Average & & & 0.347 & & & & \\
\hline
\end{tabular}

Notes: LFS -Labor Force Survey Self Emp.-Self-employment Ratio 
We rewrite this function in linear regression form in equation (2). However, for the purpose of estimation we employ probit, logit, and linear modeling strategies.

$$
\begin{gathered}
Y=\theta_{0}+\theta_{1} \Omega+\theta_{2} Z+\theta_{3} W+\sum_{i} \mu_{i} R_{i}+\epsilon \\
\operatorname{Prob}(Y=1)=\Phi\left(\alpha_{0}+\alpha_{1} \Omega+\alpha_{2} Z+\alpha_{3} W+\sum_{i} \psi_{i} R_{i}\right) \\
\operatorname{Prob}(Y=1)=\Lambda\left(\delta_{0}+\delta_{1} \Omega+\delta_{2} Z+\delta_{3} W+\sum_{i} \chi_{i} R_{i}\right)
\end{gathered}
$$

In equations (2), (3) and (4), the dependent variable is a binary indicator which takes on a value of 1 if an individual is self-employed and a value of 0 if the individual is a wage-earner. $\Phi($. in equation (3) indicates the standard normal distribution and $\Lambda($.$) in equation (4)indicates the$ logistic cumulative distribution function. Variables included in the $\Omega$ matrix are sex, education, number of children in the family, size of the family, marital status, cohort of birth, region, whether an individual lives in a metro area, if observation is a male with child, if observation is male and married, and home ownership status. $Z$ is a matrix of dummy variables including year and region dummies, and fixed effects. The vector $W$ captures the proxy for wealth. This research makes use of two wealth proxies; interest income and dividend income. As defined in the CPS, interest income captures how much pre-tax income (if any) the respondent received from interest on saving accounts, certificates of deposit, money market funds, bonds, treasury notes, IRAs, and/or other investments that pay interest. In contrast, dividend income captures returns that respondents received from stocks and mutual funds during the previous calendar year. Interest income is clearly a more broad measure and provides a better proxy of wealth, however, the dividend income wealth proxy allows examination of the robustness of our results. We estimate models using both wealth proxies and get similar results, but we focus primarily on results obtained using interest income 2 The $R$ matrix contains dummy variables that captures our break down of the population based on race,

\footnotetext{
${ }^{2}$ In Belton and Uwaifo (2008) arguments for why interest income makes a good proxy for saving/wealth are highlighted.
} 
immigration status, and for foreign born, home country economic status. $R_{1} \sim$ U.S born Black, $R_{2} \sim$ Foreign Black, $R_{3}$ American Indian/Eskimio and Aluet, $R_{4} \sim$ Foreign Asians from developed countries, $R_{5} \sim$ Foreign Asians from developing countries, $R_{6} \sim$ Naturalized Asians from developing countries, $R_{7} \sim$ Naturalized Asians from developed countries, $R_{8} \sim$ Asian-Americans, $R_{9} \sim$ Others, $R_{10} \sim$ Naturalized American Whites from less developed countries, $R_{11} \sim$ Naturalized American Whites from developed countries, $R_{12} \sim$ Naturalized American Blacks, $R_{13} \sim$ Foreign Whites from LDC (less developed or developing country), $R_{14} \sim$ Foreign Whites from DC (developed country). The vector of coefficients on the $\mathrm{R}$ matrix is the focus of our analysis. Note, in this analysis the baseline group for comparison is U.S born White Americans. Finally, $\epsilon$ is a vector of error terms. In this research the variables of interest are the $R_{5}, R_{4}, R_{7}, R_{6}, R_{10}, R_{11}, R_{2}, R_{12}, R_{13}$ and $R_{14}$. We do not partition foreign Blacks or naturalized Blacks into those from LDCs and DCs because the number of Blacks in our data set that immigrate from DCs is insignificant and does not provide a sufficiently large test sample.

We estimate equation (2), (3) and (4) the linear, probit and logit models. In addition to presenting the estimated coefficients from the all models, we also present the marginal effects from the probit estimation. These effects represent the marginal impact of an infinitesimal change in each independent continuous variable on the probability of self-employment, providing the most straight forward interpretation of estimated results from the probit models. For race dummy variables, the interpretations are slightly different as the estimates capture the marginal effect of a change in the probability of being self-employed for a particular racial group relative to the baseline group. Similarly, for ease of interpretation, we present the odds ratio using the logit model 3 The linear probability specification of the binary choice model also provides ease of interpretation. However care must be taken because, unless restrictions are placed on estimates, coefficients can imply probabilities outside the unit interval. 
Table 3: Does economic status of home country matter for immigrant self-employment?

\begin{tabular}{|c|c|c|c|c|c|c|c|c|c|c|}
\hline \multirow[t]{2}{*}{ Variable: } & \multicolumn{5}{|c|}{ Saving Indicator 1} & \multicolumn{5}{|c|}{ Savings Indicator 2} \\
\hline & $\begin{array}{c}\text { logit } \\
(1)\end{array}$ & $\begin{array}{c}\text { odds ratio } \\
(2)\end{array}$ & $\begin{array}{c}\text { Linear } \\
(3)\end{array}$ & $\begin{array}{c}\text { Probit } \\
(4)\end{array}$ & $\begin{array}{l}\text { M.E } \\
(5)\end{array}$ & $\begin{array}{c}\operatorname{logit} \\
(6)\end{array}$ & $\begin{array}{c}\text { odds ratio } \\
(7)\end{array}$ & $\begin{array}{c}\text { Linear } \\
(8)\end{array}$ & $\begin{array}{c}\text { Probit } \\
(9)\end{array}$ & $\begin{array}{l}\text { M.E } \\
(10)\end{array}$ \\
\hline logincint & $\begin{array}{c}0.091^{*} \\
(0.003)\end{array}$ & $\begin{array}{l}1.095^{*} \\
(0.004)\end{array}$ & $\begin{array}{c}0.01^{*} \\
(0.0003)\end{array}$ & $\begin{array}{c}0.048^{*} \\
(0.002)\end{array}$ & $\begin{array}{c}0.009^{*} \\
(0.0003)\end{array}$ & & & & & \\
\hline logdefdiv & & & & & & $\begin{array}{c}0.053^{*} \\
(0.005) \\
10.56\end{array}$ & $\begin{array}{c}1.05^{*} \\
(0.005) \\
10.56\end{array}$ & $\begin{array}{c}0.006^{*} \\
(0.0006) \\
10.86\end{array}$ & $\begin{array}{c}0.029^{*} \\
(0.003) \\
10.58\end{array}$ & $\begin{array}{c}0.006^{*} \\
(0.0006) \\
10.58\end{array}$ \\
\hline Black & $\begin{array}{c}-0.734^{*} \\
(0.040)\end{array}$ & $\begin{array}{c}0.48^{*} \\
(0.019)\end{array}$ & $\begin{array}{c}-0.048^{*} \\
(0.002)\end{array}$ & $\begin{array}{c}-0.359^{*} \\
(0.019)\end{array}$ & $\begin{array}{c}-0.054^{*} \\
(0.002)\end{array}$ & $\begin{array}{c}-0.796^{*} \\
(0.079)\end{array}$ & $\begin{array}{c}0.4509692 \\
(0.036)\end{array}$ & $\begin{array}{c}-0.060^{*} \\
(0.005)\end{array}$ & $\begin{array}{c}-0.396^{*} \\
(0.039)\end{array}$ & $\begin{array}{c}-0.069^{*} \\
(0.005)\end{array}$ \\
\hline BlackF & $\begin{array}{c}-0.348^{*} \\
(0.148)\end{array}$ & $\begin{array}{l}0.706^{*} \\
(0.105)\end{array}$ & $\begin{array}{l}-0.02^{*} \\
(0.008)\end{array}$ & $\begin{array}{c}-0.175^{*} \\
(0.072)\end{array}$ & $\begin{array}{c}-0.029^{*} \\
(0.010)\end{array}$ & $\begin{array}{c}-0.636^{*} \\
(0.364)\end{array}$ & $\begin{array}{l}0.529^{*} \\
(0.193)\end{array}$ & $\begin{array}{c}-0.042^{*} \\
(0.019)\end{array}$ & $\begin{array}{c}-0.347^{*} \\
(0.172)\end{array}$ & $\begin{array}{c}-0.061^{*} \\
(0.024)\end{array}$ \\
\hline $\mathrm{A} / \mathrm{A} / \mathrm{E}$ & $\begin{array}{c}-0.269^{*} \\
(0.093)\end{array}$ & $\begin{array}{l}0.766^{*} \\
(0.071)\end{array}$ & $\begin{array}{c}-0.025^{*} \\
(0.008)\end{array}$ & $\begin{array}{l}-0.13^{*} \\
(0.049)\end{array}$ & $\begin{array}{c}-0.022^{*} \\
(0.008)\end{array}$ & $\begin{array}{c}-0.319^{*} \\
(0.161)\end{array}$ & $\begin{array}{l}0.727^{*} \\
(0.117)\end{array}$ & $\begin{array}{c}-0.037^{*} \\
(0.016)\end{array}$ & $\begin{array}{c}-0.160^{* *} \\
(0.089)\end{array}$ & $\begin{array}{r}-0.032^{* *} \\
(0.016)\end{array}$ \\
\hline ForAsDC & $\begin{array}{l}0.480^{*} \\
(0.109)\end{array}$ & $\begin{array}{l}1.616^{*} \\
(0.176)\end{array}$ & $\begin{array}{l}0.052^{*} \\
(0.014)\end{array}$ & $\begin{array}{c}0.253^{*} \\
(0.06)\end{array}$ & $\begin{array}{l}0.054^{*} \\
(0.015)\end{array}$ & $\begin{array}{l}0.542^{*} \\
(0.215)\end{array}$ & $\begin{array}{l}1.72^{*} \\
(0.37)\end{array}$ & $\begin{array}{l}0.072^{*} \\
(0.034)\end{array}$ & $\begin{array}{l}0.300^{*} \\
(0.121)\end{array}$ & $\begin{array}{l}0.075^{*} \\
(0.035)\end{array}$ \\
\hline ForAsLDC & $\begin{array}{c}-0.286^{*} \\
(0.08)\end{array}$ & $\begin{array}{l}0.752^{*} \\
(0.060)\end{array}$ & $\begin{array}{c}-0.026^{*} \\
(0.006)\end{array}$ & $\begin{array}{c}-0.147^{*} \\
(0.040)\end{array}$ & $\begin{array}{c}-0.0245^{*} \\
(0.006)\end{array}$ & $\begin{array}{l}-0.156 \\
(0.140)\end{array}$ & $\begin{array}{c}0.855 \\
(0.12)\end{array}$ & $\begin{array}{l}-0.018 \\
(0.013)\end{array}$ & $\begin{array}{c}-0.084^{*} \\
(0.073)\end{array}$ & $\begin{array}{l}-0.017 \\
(0.014)\end{array}$ \\
\hline NAAsLDC & $\begin{array}{l}-0.029 \\
(0.055)\end{array}$ & $\begin{array}{c}0.971 \\
(0.054)\end{array}$ & $\begin{array}{l}-0.004 \\
(0.006)\end{array}$ & $\begin{array}{c}-0.016 \\
(0.03)\end{array}$ & $\begin{array}{l}-0.003 \\
(0.005)\end{array}$ & $\begin{array}{r}-0.137 \\
(0.09)\end{array}$ & $\begin{array}{c}0.872 \\
(0.079)\end{array}$ & $\begin{array}{c}-0.0168 \\
(0.011)\end{array}$ & $\begin{array}{l}-0.077 \\
(0.049)\end{array}$ & $\begin{array}{r}-0.016 \\
(0.01)\end{array}$ \\
\hline NAAsDC & $\begin{array}{c}0.789^{*} \\
(0.095)\end{array}$ & $\begin{array}{l}2.202^{*} \\
(0.209)\end{array}$ & $\begin{array}{c}0.110^{*} \\
(0.016)\end{array}$ & $\begin{array}{l}0.436^{*} \\
(0.055)\end{array}$ & $\begin{array}{l}0.102^{*} \\
(0.016)\end{array}$ & $\begin{array}{c}0.768^{*} \\
(0.162)\end{array}$ & $\begin{array}{c}2.16^{*} \\
(0.348)\end{array}$ & $\begin{array}{l}0.115^{*} \\
(0.029)\end{array}$ & $\begin{array}{c}0.432^{*} 3 \\
(0.095)\end{array}$ & $\begin{array}{c}0.115^{*} \\
(0.030)\end{array}$ \\
\hline AAsiann & $\begin{array}{c}-0.293^{*} \\
(0.067)\end{array}$ & $\begin{array}{c}0.746^{*} \\
(0.05)\end{array}$ & $\begin{array}{r}-0.028^{*} \\
(0.006)\end{array}$ & $\begin{array}{r}-0.152^{*} \\
(0.035)\end{array}$ & $\begin{array}{c}-0.025^{*} \\
(0.005)\end{array}$ & $\begin{array}{l}-0.30^{*} \\
(0.104)\end{array}$ & $\begin{array}{l}0.744^{*} \\
(0.077)\end{array}$ & $\begin{array}{c}-0.032^{*} \\
(0.01)\end{array}$ & $\begin{array}{r}-0.155^{*} \\
(0.054)\end{array}$ & $\begin{array}{c}-0.031^{*} \\
(0.01)\end{array}$ \\
\hline Others & $\begin{array}{c}-0.458^{*} \\
(0.148)\end{array}$ & $\begin{array}{l}0.632^{*} \\
(0.094)\end{array}$ & $\begin{array}{c}-0.04^{*} \\
(0.01)\end{array}$ & $\begin{array}{c}-0.231^{*} \\
(0.075)\end{array}$ & $\begin{array}{c}-0.037^{*} \\
(0.01)\end{array}$ & $\begin{array}{l}-0.524 \\
(0.331)\end{array}$ & $\begin{array}{c}0.592 \\
(0.196)\end{array}$ & $\begin{array}{c}-0.049^{*} \\
(0.026)\end{array}$ & $\begin{array}{c}-0.294^{* *} \\
(0.174)\end{array}$ & $\begin{array}{r}-0.054^{* *} \\
(0.026)\end{array}$ \\
\hline NAWLDC & $\begin{array}{c}0.146^{*} \\
(0.041)\end{array}$ & $\begin{array}{l}1.157^{*} \\
(0.048)\end{array}$ & $\begin{array}{l}0.016^{*} \\
(0.005)\end{array}$ & $\begin{array}{c}0.078^{*} \\
(0.023)\end{array}$ & $\begin{array}{l}0.015^{*} \\
(0.005)\end{array}$ & $\begin{array}{c}0.106 \\
(0.076)\end{array}$ & $\begin{array}{c}1.11 \\
(0.085)\end{array}$ & $\begin{array}{l}0.014 \\
(0.01)\end{array}$ & $\begin{array}{c}0.063 \\
(0.043)\end{array}$ & $\begin{array}{l}0.014 \\
(0.01)\end{array}$ \\
\hline NAWDC & $\begin{array}{c}0.369^{*} \\
(0.054)\end{array}$ & $\begin{array}{c}1.45^{*} \\
(0.077)\end{array}$ & $\begin{array}{l}0.051^{*} \\
(0.008)\end{array}$ & $\begin{array}{c}0.208^{*} \\
(0.030)\end{array}$ & $\begin{array}{l}0.043^{*} \\
(0.007)\end{array}$ & $\begin{array}{c}0.340^{*} \\
(0.088)\end{array}$ & $\begin{array}{c}1.41^{*} \\
(0.123)\end{array}$ & $\begin{array}{l}0.053^{*} \\
(0.015)\end{array}$ & $\begin{array}{l}0.198^{*} \\
(0.050)\end{array}$ & $\begin{array}{c}0.047^{*} \\
(0.013)\end{array}$ \\
\hline NAB & $\begin{array}{c}-0.310^{*} \\
(0.133)\end{array}$ & $\begin{array}{l}0.734^{*} \\
(0.097)\end{array}$ & $\begin{array}{c}-0.027^{*} \\
(0.01)\end{array}$ & $\begin{array}{r}-0.163^{*} \\
(0.068)\end{array}$ & $\begin{array}{c}-0.027^{*} \\
(0.01)\end{array}$ & $\begin{array}{l}-0.289 \\
(0.259)\end{array}$ & $\begin{array}{c}0.748 \\
(0.194)\end{array}$ & $\begin{array}{l}-0.028 \\
(0.023)\end{array}$ & $\begin{array}{l}-0.129 \\
(0.139)\end{array}$ & $\begin{array}{l}-0.026 \\
(0.026)\end{array}$ \\
\hline WFLDC & $\begin{array}{c}0.018 \\
(0.048)\end{array}$ & $\begin{array}{c}1.018 \\
(0.049)\end{array}$ & $\begin{array}{l}0.0002 \\
(0.004)\end{array}$ & $\begin{array}{c}0.01 \\
(0.025)\end{array}$ & $\begin{array}{c}0.002 \\
(0.005)\end{array}$ & $\begin{array}{c}0.137 \\
(0.119)\end{array}$ & $\begin{array}{c}1.146 \\
(0.136)\end{array}$ & $\begin{array}{c}0.014 \\
(0.013)\end{array}$ & $\begin{array}{c}0.073 \\
(0.064)\end{array}$ & $\begin{array}{c}0.016 \\
(0.015)\end{array}$ \\
\hline WFDC & $\begin{array}{c}0.352^{*} \\
(0.061)\end{array}$ & $\begin{array}{c}1.42^{*} \\
(0.087)\end{array}$ & $\begin{array}{c}0.04^{*} \\
(0.008)\end{array}$ & $\begin{array}{c}0.190^{*} \\
(0.034)\end{array}$ & $\begin{array}{l}0.039 * \\
(0.008)\end{array}$ & $\begin{array}{l}0.336^{*} \\
(0.103)\end{array}$ & $\begin{array}{c}1.40^{*} \\
(0.145)\end{array}$ & $\begin{array}{l}0.044^{*} \\
(0.015)\end{array}$ & $\begin{array}{l}0.183^{*} \\
(0.059)\end{array}$ & $\begin{array}{c}0.043^{*} \\
(0.015)\end{array}$ \\
\hline Constant & $\begin{array}{c}-2.64^{*} \\
(0.238)\end{array}$ & & $\begin{array}{c}0.114^{*} \\
(0.03)\end{array}$ & $\begin{array}{c}-1.51^{*} \\
(0.13)\end{array}$ & & $\begin{array}{l}-2.92^{*} \\
(0.411)\end{array}$ & & $\begin{array}{l}0.083^{*} \\
(0.055)\end{array}$ & $\begin{array}{l}-1.66^{*} \\
(0.224)\end{array}$ & \\
\hline
\end{tabular}

Controls: age, $a g e_{2}$, education, number of children, owning a home, head of household, male with child, male married, family size, metro area, cohort, region, year dummies, martial status, gender.

Variable descriptionA/A/E American Indian/Aleut/Eskimo; NAB-Naturalized American Black; BlackFBlack foreign; WFLC- White foreign less developing countries, FWDC- White foreign developed countries; race3-Native American,Aleut and Alaskan; NAWLDC-Naturalized White LDC; NAWDC-Naturalized White from DC; AAsian-U.S born Asian or Pacific Islander(PI); NAAsLDC-Naturalized Asian/PI from LDC; NAAsDC -Naturalized Asian/PI from DC; ForAsLDC-foreign Asians/PI from LDC; ForAsDC-foreign Asian/PI from DC. Column (1)-(5) control for resource using interest income and columns (6) to (10) controls for resource using dividend income. 10 M.E-Marginal Effects 


\subsection{Estimation Results}

Table (3) provides linear, logit, probit, and probit marginal effects estimation results of equation (1). We focus primarily on marginal effects results in columns (5) and (10). Our results show that foreign Blacks have a $2.9 \%$ lower probability of being self-employed than native born White Americans. Since most foreign Blacks in the U.S are immigrants from developing countries, the coefficient associated with foreign Blacks represent a direct comparison of Blacks from developing countries to U.S. born White Americans.

In the case of Asians, we find that foreign Asians from less developed countries (LDCs) have a $2.5 \%$ lower probability of self-employment than native born White Americans, while foreign Asians from developed countries (DCs) have a $5.4 \%$ higher probability of being self-employed than native born White Americans. The difference between foreign Asians immigrants from developed and developing countries is significant as foreign Asians from developed countries are over $7 \%$ more likely to be self-employed than are their counterparts from developing countries. Previous research has offered various explanations for the high rates of Asian self-employment including high levels of human and financial capital, rotating credit associations, and access to co-ethnic labor and customers(See Bates (1997), Zhou (2004) and Light (2004)). However, our results show that not all Asians enjoy these advantages. Foreign Asians from less developed countries have similar probabilities of self-employment to that of foreign Blacks from less developed countries. This finding makes the argument of disproportionate Asian self-employment success somewhat less tenable.

Examining the home country's economic status impact for Whites, we find similar results as in the case of Asians. Foreign White immigrants from developing countries have a similar probability of self-employment to that of White Americans while foreign White immigrants from developed countries have a 3.9\% higher probability of self-employment than do native born White Americans. These results show that consistent across race, foreigners from developed countries have a higher probability of self-employment than do those from developing countries, while those from developing countries had lower or equal probability of self-employment to that of U.S. born Whites. This result

\footnotetext{
${ }^{3}$ The odds ratio are the exponentiated coefficients in an ordinary logistic regression.
} 
may suggest that an immigrants home country's economic status matters for self-employment.

\subsection{Robustness}

Results above reveal that self-employment probabilities differs among foreign immigrant groups based on home country economic status. However, to test the robustness of our home country's economic status impact, we examine self-employment trends among naturalized American immigrants. Naturalized Americans are immigrants who have been able to successfully change their citizenship status over time. Looking again at the marginal effects in columns (5) and (10) and focusing first on naturalized Asians from LDCs. We find that their self-employment probability is identical to that of U.S born Whites, while their counterparts, naturalized Asians from DCs have a 10\% higher

probability of self-employment than U.S. born Whites. Once again the developed country effect is significant for naturalized Asians as the gap between the self-employment probabilities for naturalized Asians from developed countries versus developing countries is larger than is this difference for foreign Asian immigrants.

As in the Asian case, we also examine the home country's economic status impact on selfemployment probabilities for naturalized Whites. We find that naturalized White Americans from less developed countries have a 1.5\% higher probability of being self-employed than U.S. born Whites while their comparable counterparts, naturalized Whites from developed countries have a $4.3 \%$ higher probability of being self-employed than U.S. born Whites. Clearly, empirical results show that across race and citizenship status immigrant home country level of economic development impacts the probability of self-employment.

As a further test of the validity of the results, we follow the approach of Fairlie and Meyers (1996) and reestimate the model using only a sample for which home country's economic status effect is relevant. We drop all Americans from the data set and estimate equation (1) using a sample of non-citizens. In this case the $\mathrm{R}$ variable is a dummy indicating home country economic status, where developed home country $=1$ and less developed home country $=0$. The results of this analysis is summarized in Table (4). Column (1) summarize results from the logit model using both interest income and dividend income as wealth proxies. Column (2) shows the marginal effects of 
the probit model using both wealth proxies. The inclusion of dividend income leads to a fall in the number of sample observations because only a few survey participants report dividend income holdings. Column (3) reveals that excluding dividend income and using only interest income as a wealth proxy does not change results significantly. We find that immigrants from developed countries have a $4 \%$ higher probability of being self-employed in the U.S. than do immigrants from developing countries. This result is similar to that obtained when the entire data set is used in model estimation. Recall that there was a $7 \%$ point difference between foreign Asians from DCs and their counterpart from LDCs and a $4 \%$ point difference between foreign White Americans from LDC and foreign White Americans from DCs. The dummy variable in this case is a mean of effects of foreign Asians and foreign Whites from developed countries.

\section{Explaining the Developed Country Effect}

We explore two likely explanations for the result that immigrants from developed countries have higher probability of self-employed in the U.S. than do immigrants from developing countries. We examine a resources availability argument and an economic institutional difference argument, however, we can not conclude with significant confidence that these two arguments provide the only explanations for our empirical result.

\subsection{The Resource Explanation}

Bogan and Darity (2008) highlight the resource availability issue in self-employment decision, arguing that many immigrants have assess to resources from their home country making self-employment success more likely. Resource endowments could differ across developed and less developed countries as foreigners from less developed countries are less likely to have tangible capital resources and assets that can be transferred to the U.S. Hence, if resource availability affects entry into self-employment, then according to the Bogan and Darity (2008) argument, foreigners from less developed countries should have a lower probability of self-employment than do those from developed countries. If the explanation for the gap is resources, then controlling for resources in our probability model should help to explain the developed versus developing country gap. Results summarized in Tables (2) 
Table 4: Robustness Checks: Does home country economic status matter for immigrant selfemployment?

\begin{tabular}{cccc}
\hline \hline & \multicolumn{3}{l}{} \\
\cline { 2 - 4 } & $\begin{array}{c}\text { logit } \\
(1)\end{array}$ & $\begin{array}{c}\text { marginal effects } \\
(2)\end{array}$ & $\begin{array}{c}\text { marginal effects } \\
(3)\end{array}$ \\
\hline Developed Home Country & $0.323^{*}$ & $0.0387^{*}$ & $0.0407^{*}$ \\
& $(0.138)$ & $(0.016)$ & $(0.007)$ \\
log saving proxy & 0.046 & 0.005 & 0.007 \\
log dividend & $(0.037)$ & $(0.004)$ & $(0.001)$ \\
& 0.035 & 0.005 & \\
education & $(0.035)$ & $(0.004)$ & \\
& $-0.0478^{* *}$ & $-0.006^{*}$ & 0.0002 \\
age & $(0.026)$ & $(0.003)$ & $(0.0008)$ \\
sex & 0.155 & 0.015 & 0.005 \\
& $(0.113)$ & $(0.012)$ & $(0.004)$ \\
no. of children & $-1.18^{*}$ & $-0.12^{*}$ & $-0.064^{*}$ \\
& $(0.33)$ & $(0.036)$ & $(0.0117)$ \\
own a home & 0.006 & -0.004 & 0.006 \\
& $(0.128)$ & $(0.014)$ & $(0.004)$ \\
family size & $0.524^{*}$ & $0.0557^{*}$ & $0.044^{*}$ \\
Head & $(0.172)$ & $(0.017)$ & $(0.006)$ \\
& -0.083 & -0.007 & $-0.006^{*}$ \\
male married & $(0.095)$ & $(0.01)$ & $(0.0027)$ \\
& $0.449^{*}$ & $0.0492^{*}$ & $0.017^{*}$ \\
male with child & $(0.156)$ & $(0.017)$ & $(0.006)$ \\
& $-0.997^{*}$ & $-0.1^{*}$ & $-0.034^{*}$ \\
No of obs. & $(0.396)$ & $(0.042)$ & $(0.014)$ \\
& 0.0624 & 0.012 & -0.006 \\
& $(0.25)$ & $(0.029)$ & $(0.01)$ \\
& 2404 & 2404 & 15129 \\
\hline \hline
\end{tabular}

Controls: age, $a_{g} e_{2}$, education, number of children, owning a home, head of household, male with child, male married, family size, metro area, cohort, region, year dummies, martial status, gender.

Variable description Columns (1) and (2) control for resource using interest income and dividend income. The sample of those with no dividend income is substantial and explains the drop in number of observations. See column(3) where wealth is only controlled for using savings the sample size is much larger. 
and (3) reveal that after controlling for resource availability using two wealth indicators the gap remains. The persistence of the gap does not imply that the results cannot be explained with a resource argument but may point to limitation in capturing wealth and/or savings resources for foreigners. Belton and Oyelere (2008) point out that the reliability of immigrant financial resource data maybe questionable given that many U.S. immigrants maintain financial relationships in their home country and tend to engage in significant remittance to the home country. This behavior suggests that savings and/or wealth of immigrants are less likely to be captured with accuracy in U.S. data sources. Data on remittances from the World Bank highlights the top 10 remittance recipients in 2007 for developing countries. They are: India ( $\$ 27.0$ bn), China ( $\$ 25.7$ bn), Mexico ( $\$ 25.0$ bn), Philippines ( $\$ 17.0$ bn), Romania ( $\$ 6.8$ bn), Bangladesh ( $\$ 6.4$ bn), Pakistan ( $\$ 6.1$ bn), Indonesia ( $\$ 6.0$ bn), Egypt, Arab Rep. ( $\$ 5.9$ bn), and Morocco ( $\$ 5.7$ bn). Remittances flowing to less developed countries are substantial however, developed countries are also recipients of significant amounts of remittances. The top 10 in 2007 are: France $(\$ 12.5$ bn), Spain ( $\$ 8.9$ bn), Belgium (\$7.2 bn), United Kingdom (\$7.0 bn), Germany ( $\$ 7.0$ bn), Portugal ( $\$ 3.8$ bn), Austria (\$3.5 bn), Australia ( $\$ 3.1 \mathrm{bn})$, United States $(\$ 3.0 \mathrm{bn})$, and Italy $(\$ 2.6 \mathrm{bn})$. It is interesting to note that significant remittances flow from developed to developing countries and from developed to developed countries. However, given home country economic conditions it is more likely that remittance to developing countries represent consumption subsides to developing country families. In contrast, remittance to developed countries is more likely for savings and investment purposes rather than a consumption subsidy. This difference in remittance could have a direct impact on our empirical analysis. Our estimates for access and level of capital and/or wealth resources for all immigrants is likely to be underestimated, however, we are more likely to underestimate this difference for those from developed countries rather than those from developing countries. Hence, measurement error in our control for wealth could be, in part, responsible for the remaining self-employment gap between immigrants from developed relative to less developed countries. 


\subsection{The Institution and Information explanation}

An alternative explanation for this result is the role of institutions and information. It is important to note that even if resource availability is the same for individual immigrants across developed and less developed countries, institutional development and the ability to access informational resources could explain the developed country effect. Recall that we have controlled, in some form, (directly or through proxy variables) for all the likely candidate that impact self-employment, however, we are not able to control for institutional similarity. For example, banking and communications systems operate in a similar fashion across developed countries. In addition, institutional, technological, and logistical similarities suggest that businesses form and operate similarly in developed countries but very differently than those in developing countries. Further, skill levels needed to operate a small business in LDCs may be significantly different than those needed in developed countries. In addition, the logistics needed to start a business could be very different between developing and developed countries, but similar across developed countries. The institutional and developmental similarities across developed countries could provide immigrants from a developed country a significant information advantage over those from LDCs in their effort to pursue self-employment in the U.S. Hence, we argue that the more similar are U.S. institutions to an immigrant's home country the more quickly immigrants are able to assimilate and get involved in U.S. self-employment activities. Recall that North (1973) defines institutions as the formal and informal rules that guide social interactions. Moreover, if in the entrepreneurship arena, institutions in the North sense and business "know how" are similar across developed countries but are significantly different relative to developing countries, then the results found in this paper are expected. In summary, though immigrants from LDCs have more historical exposure to self-employment relative to immigrants from DCs, given, potential difference in experiences and different information sets for LDC immigrants, DC immigrants when all prior conditions are considered, may find it easier to transition into self-employment in the U.S.

Though we cannot directly test the institutional hypothesis, we know that institutional impacts are long-lived and that it takes time to acquire new skill sets, institution specific business "know 
how", and acquire information. Our results show a persistence in the gap in self-employment between immigrants from developed countries relative to those from developing countries, even after naturalization 4 This result is compatible with institutional and/or informational explanations for the gap.

\subsection{Evidence For Institutions and Against Resource}

Interestingly, the results in Table (2) provides suggestive evidence in support of the information and institution argument. In addition the results highlight why resource differences may not fully explain our research results. The possibility that there are alternative explanations for the gap is reflected in the differential behavior noted when comparing naturalized citizens to foreign immigrants from DCs relative to LDCs. Naturalized citizen unlike foreigners have full access to the U.S financial systems. Given that naturalized citizens are not legally constrained, access to capital is in all likelihood less difficult than for their foreign counterparts. If we assume that the resource constraint is binding for all immigrants, leading to a reduction in self-employment success, then when immigrants become naturalized we should see similar increases in self-employment as this constraint is relaxed. This result implies that coefficients differences between naturalized relative to foreign immigrants across race and home country economic status should be similar. The results in Table (2), however, do not support this outcome. We note that there is a $1.5 \%$ point higher probability of self-employment for naturalized Whites from LDCs relative to foreign Whites from LDCs. This increase is larger than the $0.4 \%$ point increase when comparing naturalized Whites from DCs to foreign Whites from DCs. In contrast, we find a $2.45 \%$ point higher probability of self-employment for naturalized Asian from LDCs in comparison to foreign Asian from LDCs. This difference is smaller than the $4.8 \%$ point higher probability of self-employment when comparing naturalized Asians from DCs to foreign Asians from DCs. Further, comparing naturalized Blacks to foreign Blacks, who are in most cases from developing countries, we find a $0.2 \%$ increase in self-employment probabilities, although this difference is not significantly different from zero. The lack of consistency across race suggest that

\footnotetext{
${ }^{4} 3$ years is the least time it takes to become a citizen which occurs if one marries a U.S citizen. Typically, it take at least 5 years from becoming a permanent resident of the U.S.
} 
the resources availability may not be the only mechanism impacting self-employment decision for immigrants from both developed and developing countries 5 In addition, the lack of increase in selfemployment for naturalized Blacks and lower increase for naturalized Asians from LDCs relative to those form DCs, provides evidence in support of our institution/information argument. The impact of institutions are long-lived and becoming a naturalized citizen though it may reduces resource restriction does not eliminate the issues of skill miss-matches and other problems highlighted above that immigrants from LDC's are more likely to face.

\section{Conclusion and Recommendations}

This research finds that home country's economic status matters for self-employment of immigrants. Specifically across race, immigrants from developed countries tend to engage in self-employment at a higher rate than all survey participants. Data errors in measurement of wealth/financial resources could account for this developed country advantage i.e., the economic development proxy used here could provide an additional wealth or financial resource measure. Conversely, the developed country effect could represent a proxy for institutional and development infrastructure of the country of origin, which when similar to the U.S provides for easy assimilation into self-employment. We do not find evidence that immigrants from countries with high levels of self-employment have higher levels of self-employment in the U.S. This again may points to the institutional explanation that despite high self-employment in the home country, if institutions are not similar, the transfer of the skill and knowledge might be slower despite an individuals exposure to self-employment in the past.

This research is the first to examine the impact of immigrants home country economic status on the probability of self-employment, and our results are at variance with the literature which suggests that immigrants are more likely to be entrepreneurs if immigrating from countries with high levels of self-employment. We care about this result because entrepreneurship is one of the drivers of economic growth and immigration has become important to U.S labor force growth. There

\footnotetext{
${ }^{5}$ If we assume resources were not a binding constraint for those from developed countries then all thing being equal we should see a smaller increase in self-employment as individuals transition from foreign to Naturalized if home country is a developed country in comparison to those from developing countries. The results for White immigrants fits this trend but the results for Asians and Blacks runs counter to this trend which again highlights other possible explanations for the results.
} 
is some evidence that suggests that immigrants from developing countries are more likely to exploit U.S. social welfare programs than are those from developed countries. Given the increase in the number of immigrants from LDCs relative to DCs, then understanding the factors that impact self-employment may lead to better policy outcomes. Many immigrants, particularly those from non-English speaking countries, have difficulty making the transition into the formal labor sector because in many instances skills and education are not easily transferable. For these immigrants, self-employment becomes one possible mechanism for assimilation and eventual movement into the U.S. work force. Understanding barriers to assimilation and other issues that lead to a slower than expected integration into U.S. economic life becomes a prerogative. We hope to focus future research on trying to disentangle the impact of capital resource access from differences in institutional framework on assimilation into self-employment. 


\section{References}

[1] Bates, Timothy, 1987 "Self-Employed Minorities: Traits and Trends," Social Sciences Quarterly68 (Sept.1987), 539-550.

[2] Bates, Timothy. 1997. Race, Self-Employment \& Upward Mobility: An Illusive American Dream, Washington, D.C.: Woodrow Wilson Center Press and Baltimore: John Hopkins University Press.

[3] Belton, Willie \& Uwaifo Oyelere, Ruth, 2008. "The Racial Saving Gap Enigma: Unraveling the Role of Institutions," IZA Discussion Papers 3545, Institute for the Study of Labor (IZA).

[4] Blanchflower, David G. \& Phillip B. Levine \& David J. Zimmerman, 2003. "Discrimination in the Small-Business Credit Market," The Review of Economics and Statistics.

[5] Blanchflower, David G. and Andrew J. Oswald. 1998. "What Makes an Entrepreneur?" Journal of Labor Economics, 1998, 16, 26-60.

[6] Blau, Francine D \& Graham, John W, 1990. "Black-White Differences in Wealth and Asset Composition," The Quarterly Journal of Economics, MIT Press, vol. 105(2), pages 321-39, May

[7] Borjas, George J \& Bronars, Stephen G, 1989. "Consumer Discrimination and Selfemployment," Journal of Political Economy, University of Chicago Press, vol. 97(3), pages 581-605, June.

[8] Bogan, Vicki and William Darity. 2008. "Culture and Entrepreneurship? African-American and Immigrant Self-Employment in the United States," The Journal of Socio-Economics forthcoming 2008

[9] Briggs, Vernon JM. and Stephen Moore. 1994, "Still the Open Door? U.S. Immigration Policy and the American Economy." Washington, DC: American University Press.

[10] Fairlie, Robert W. 1999. "The Absence of the African-American Owned Business: An Analysis of the Dynamics of Self-Employment." Journal of Labor Economics, 17(1): 80- 108. 
[11] Fairlie, Robert W., and Bruce D. Meyer. 1996. "Ethnic and Racial Self-Employment Differences and Possible Explanations." Journal of Human Resources 31(4):757-793.

[12] Fairlie, R. W., \& Meyer, B. D. 2000. ?Trends in Self-Employment Among White and Black Men During the Twentieth Century.? The Journal of Human Resources, 35 (4), p. 643-669.

[13] Fairlie, Robert W. and Alicia M. Robb 2007 "Why Are Black-Owned Businesses Less Successful than White-Owned Businesses? The Role of Families, Inheritances, and Business Human Capital". Journal of Labor Economics, 2007, vol. 25, issue 2, pages 289-324.

[14] Kotkin, Joel. September 1991. "The American Way." Inc. p.96

[15] Laband, D. and B.F. Lentz. 1990. "Entrepreneurial success and occupational inheritance among proprietors". Canadian Journal of Economics, 23(3):101-117.

[16] Light,Ivan. 2004. "The Ethnic Economy." In Neil Smelser and Richard Swedbergs, eds., Hamdbook in Economic Sociolgy (2n ed.). New York: Russell Sage Foundation.

[17] Magnus Lofstrom, Timothy Bates. 2007. "African Americans? Pursuit of Self-Employment" IZA Working Paper DP3156.

[18] Meyer, Bruce. 1990. "Why Are There So Few Black Entrepreneurs?" National Bureau of Economic Research, Working Paper No. 3537.

[19] Miriam King, Steven Ruggles, Trent Alexander, Donna Leicach, and Matthew Sobek. Integrated Public Use Microdata Series, Current Population Survey: Version 2.0. [Machine-readable database]. Minneapolis, MN: Minnesota Population Center [producer and distributor], 2004.

[20] North, Douglass C. and Robert Paul Thomas (1973), The Rise of the Western World: A New Economic History. New York: Cambridge University

[21] Smith, J. P. and Welch, F. R. "Black Economic Progress After Myrdal," Journal of Economic Literature, June 1989, 27 (2), pp. 519-64. 
[22] Yuengert, Andrew M. 1995 "Testing Hypotheses of Immigrant Self-Employment." Journal of Human Resources. Vol. 30 No. 1 pp. 194-204.

[23] Zhou, M. 2004. "Revisiting Ethnic Entrepreneurship: Convergencies, Controversies, and Conceptual Advancement." International Migration Review 38(3): pp. 1040-1074. vspace40mm 